\title{
Robust Servo-system Based on Two-Degree- -of-Freedom Control with Sliding Mode
}

\author{
Yasutaka Fujimoto, Student Member, IEEE, Atsuo Kawamura, Member, IEEE
}

\begin{abstract}
A robust servo-system, based on a combination of linear robust control and sliding mode control have been proposed. This new control system can be said to be a non-linear system (sliding mode control system) which has a inner loop of linear control (two-degree-of-freedom control). Due to this inner loop of linear control, a disturbance is strongly suppressed but not completely. Then, the outer loop of sliding mode control eliminates this disturbance suppression error.

First in this paper, a linear robust-servo design of twodegree-of-freedom control system is shown. Next, a sliding mode control is applied to this system and the disturbance suppression characteristics are discussed. Then, through the simulations and experiments, it is proved that the introduction of non-linear control (sliding mode) drastically improves the disturbance suppression characteristics of a linear system (two-degree-of-freedom control).
\end{abstract}

\section{INTRODUCTION}

In the field of robot manipulator control, one of the important issues is robustness of the servo-system. The robustness of a servo-system applied to each joint of a manipulator enables non-interferences among other joints. Several papers have been published concerning this issue [10][15].

Particularly, the authors have proposed such a robust servo-system, based on a combination of disturbance observer and sliding mode control [13]-[15]. From the viewpoint of VSS, this control law has an advantage in the robustness with the less chattering, even at a relatively low sampling frequency. The introduction of the disturbance observer improves the robustness of conventional sliding mode control.

However, some papers show that very robust system against a disturbance and a fluctuation of plant parameters can be constructed using only the disturbance observer [9], [10], with a structure of two-degree-of-freedom control system. In other words, the disturbance response can be set irrelevantly to command input response. Essentially, twodegree-of-freedom control system is derived from stability and achievable transfer characteristics of a linear system [2]-[4]. As a result, it includes equivalently linear control system such as a disturbance observer [1], [5].

These linear robust controller can compensate the disturbance strongly, but not completely. There is a remaining disturbance which cannot be completely compensated by such a linear robust controller, because the cut-off frequency of disturbance reduction HPF (sensitivity function)

Manuscript received April 4, 1994; revised August 21, 1994.

The authors are with the Department of Electrical and Computer Engineering, Yokohama National University, Hodogaya-ku, Yokohama 240 Japan.

IEEE Log Number 9410545. cannot be set much high. There is an alternative trade-off between the reduction of the disturbance and the reduction of the influence of the sensor noise or the parameter fluctuation. In this paper, sliding mode control is introduced into a linear system controlled by two-degree-of-freedom controller in order to eliminate the disturbance suppression error. In the simulations and experiments, this control scheme is applied to DC servo motor positioning control, and the effectiveness is clarified.

\section{Plant Model}

In order to inspect the proposed servo control scheme, the voltage controllable DC motor is adopted as a plant. The mechanical dynamics and circuit dynamics are expressed as follows:

$$
\begin{gathered}
J \ddot{\theta}+D \dot{\theta}+T_{l}=T_{m} \\
K_{E} \dot{\theta}+L_{a} \frac{d I_{a}}{d t}+R_{a} I_{a}=u \\
T_{m}=K_{T} I_{a}
\end{gathered}
$$

where

$$
\begin{array}{ll}
\theta & \text { position }[\mathrm{rad}], \\
T_{l} & \text { load torque }[\mathrm{N} \cdot \mathrm{m}], \\
T_{m} & \text { torque generated by motor }[\mathrm{N} \cdot \mathrm{m}], \\
I_{a} & \text { rotor current }[\mathrm{A}], \text { and } \\
u & \text { dc voltage of the motor terminal }[\mathrm{V}] .
\end{array}
$$

The other parameters and the motor's specifications are shown in Table I.

Because the electrical time constant is much smaller than the mechanical one, the delay of electrical response can be ignored. At this time the following equation is derived from (1) $-(3)$ :

$$
\ddot{\theta}=-\frac{B}{J} \dot{\theta}+\frac{K}{J} u-\frac{1}{J} T_{l}
$$

where

$$
B=\frac{K_{E} K_{T}+R_{a} D}{R_{a}}, \quad K=\frac{K_{T}}{R_{a}}
$$

The plant system can be regarded as a second order system.

\section{Two-Degree-of-Freedom Control}

The characteristics of this control law is that the command input response and the disturbance response can be set independently from each other, and the stability is guaranteed [2]-[4]. Moreover this system includes conventional linear control systems such as a disturbance observer [1], [5]. Two-degree-of-freedom control system has an advantage over those systems, because it is designed positively 
TABLE I

Motor Parameters (Including Harmonic Gear)

\begin{tabular}{lccc}
\hline Rated Output Power & & $\mathrm{W}$ & 17 \\
Rated Torque & & $\mathrm{N} \cdot \mathrm{m}$ & 5.29 \\
Rated Voltage & & $\mathrm{V}$ & 24 \\
CEMF Const. & $K_{E}$ & $\mathrm{~V} / \mathrm{rad} / \mathrm{sec}$ & 5.44 \\
Torque Const. & $K_{T}$ & $\mathrm{~N} \cdot \mathrm{m} / \mathrm{A}$ & 5.44 \\
Motor Inertia & $J$ & $\mathrm{Kg} \cdot \mathrm{m}^{2}$ & 0.0974 \\
Friction Coefficient & $D$ & & - \\
Mechanical Time Const. & & $\mathrm{sec}$ & 0.056 \\
Rotor Resistance & $R_{a}$ & $\Omega$ & 2.8 \\
Rotor Inductance & $L_{a}$ & $\mathrm{mH}$ & 1.1 \\
Electrical Time Const. & & $\mathrm{msec}$ & 0.39 \\
Gear Ratio & $K_{G}$ & & 0.01 \\
\hline
\end{tabular}

using independence of the command input response and the disturbance response. Thus, the design method is very refined.

The configuration of this system is shown in Fig. 1(a). Here, $u$ is the controller's output, $r$ is the command input, $y$ is the plant's output, $d$ is the disturbance and $\xi$ the observation noise. In Fig. 1(a), the command input response $G_{y r}(s)$ and the sensitivity function $S(s)$ become

$$
\begin{aligned}
G_{y r}(s) & =\frac{y(s)}{r(s)}=\frac{P(s) C_{1}(s)}{1+P(s) C_{2}(s)} \\
S(s) & \equiv \frac{\partial G_{y r}(s) / G_{y r}(s)}{\partial P(s) / P(s)}=\frac{1}{1+P(s) C_{2}(s)} .
\end{aligned}
$$

The sensitivity function $S(s)$ indicates not only sensitivity to fluctuation of plant parameters, but also disturbance reduction characteristics.

From these equations, the following controllers are obtained:

$$
\begin{aligned}
C_{1}(s) & =\frac{G_{y r}(s)}{P_{n}(s) S(s)} \\
C_{2}(s) & =\frac{1-S(s)}{P_{n}(s) S(s)}
\end{aligned}
$$

where $P_{n}(s)$ is the nominal plant model and $G_{y r}(s), S(s)$ are the free parameters.

This expression of controllers means that the command input response and the sensitivity function can be set at the designer's will. Thus, the global system can be designed insensitive to disturbance and fluctuation of plant parameters.

However, free parameters $G_{y r}(s)$ and $S(s)$ should be selected to satisfy some conditions. The reason is that only when all transfer functions from all input signal $\{r, d, \xi\}$ to all sub-system's output $\{u, y\}$ are stable, the global system is stable. These transfer functions become

$$
\left[\begin{array}{l}
u(s) \\
y(s)
\end{array}\right]=\left[\begin{array}{ccc}
\frac{G_{y r}(s)}{P_{n}(s)} & 1-S(s) & -\frac{1-S(s)}{P_{n}(s)} \\
G_{y r}(s) & -P_{n}(s) S(s) & -(1-S(s))
\end{array}\right]\left[\begin{array}{c}
r(s) \\
d^{\prime}(s) \\
\xi(s)
\end{array}\right]
$$

where

$$
d^{\prime}(s) \equiv d(s)+\left(\frac{1}{P(s)}-\frac{1}{P_{n}(s)}\right) y(s)
$$

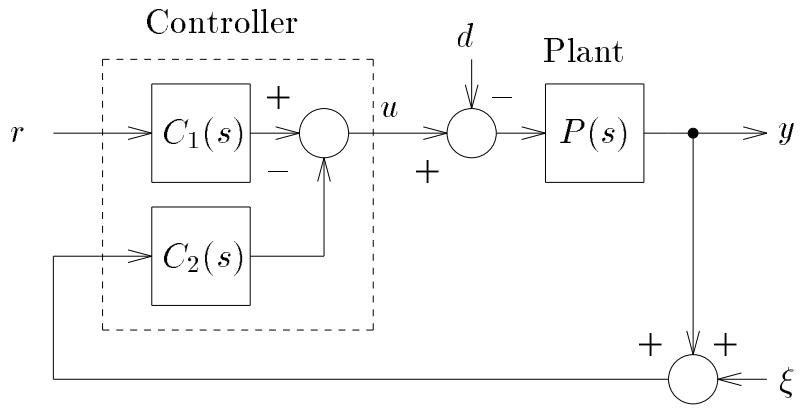

(a)

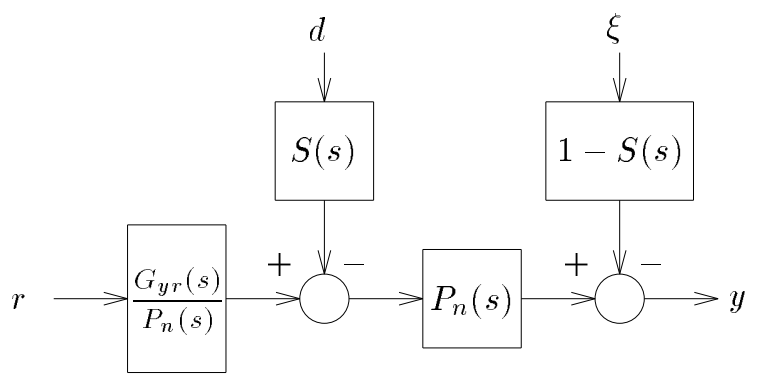

(b)

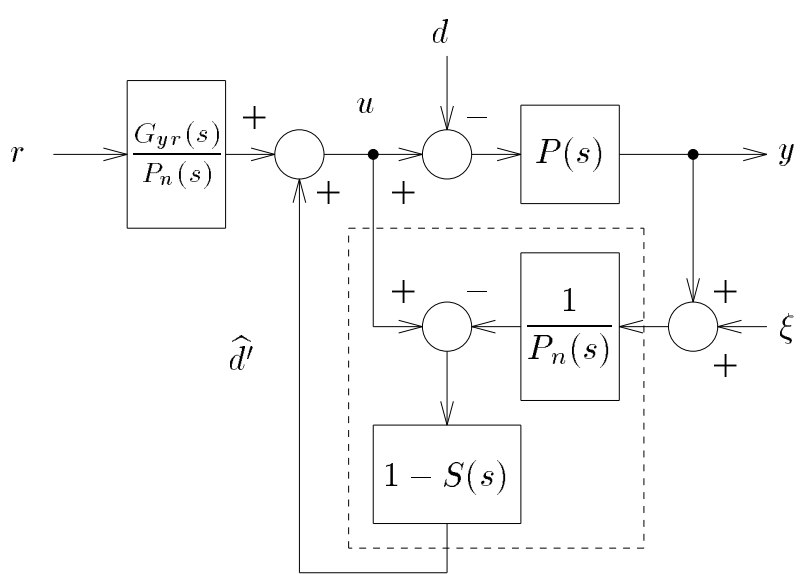

(c)

Fig. 1. Two-degree-of-freedom control system. (a) System configuration. (b) Equivalent block diagram. (c) Disturbance observer type equivalent block diagram.

$d^{\prime}$ is defined as the total disturbance which includes fluctuation of plant parameters. These transfer functions must be proper and have only stable poles.

$$
\begin{array}{ll}
\frac{G_{y r}(s)}{P_{n}(s)}, & 1-S(s), \\
\frac{1-S(s)}{P_{n}(s)}, & G_{y r}(s), \quad P_{n}(s) S(s) \quad \in R H_{\infty} .
\end{array}
$$

Here, $R H_{\infty}$ expresses a set of proper and stable transfer functions.

Moreover, the following conditions should be satisfied for 
output regulation $\left.(r-y) \rightarrow 0\right|_{t \rightarrow \infty}$.

$$
\begin{aligned}
& {\left[1-G_{y r}(s)\right] r(s),} \\
& P_{n}(s) S(s) d^{\prime}(s), \quad[1-S(s)] \xi(s) \in R H_{\infty} .
\end{aligned}
$$

From the conditions $(11),(12)$, free parameters $G_{y r}(s)$ and $S(s)$ should be selected to satisfy the following conditions:

- $G_{y r}(s)$ must be a proper and stable rational function

- $G_{y r}(s)$ must have unstable zero (include infinity) of plant as zero

- $1-G_{y r}(s)$ must have unstable pole of input signal as zero

- $1-S(s)$ must be a proper and stable rational function

- $1-S(s)$ must have unstable zero (include infinity) of plant and unstable pole of observation noise as zero

- $S(s)$ must have unstable pole of plant and disturbance as zero

Fig. 1(b) shows equivalent block diagram of Fig. 1(a) with controller $(7),(8)$. Then, the next dynamics of the total system is obtained

$$
y(s)=G_{y r}(s) r(s)-P_{n}(s) S(s) d^{\prime}(s)-[1-S(s)] \xi(s) .
$$

In order to construct the robust servo-system, parameter $S(s)$ is set to a low gain in the low-frequency domain, because fluctuation of the disturbance and the plant parameters are usually slow. And $1-S(s)$ is selected as a lowpass filter, because observation noise $\xi$ is usually at high frequency.

For example, in an application to positioning system of DC servo motor, the parameters can be set as follows:

$$
\begin{aligned}
P_{n}(s) & =\frac{K_{n}}{\left(J_{n} s+B_{n}\right) s} \\
G_{y r}(s) & =\frac{1}{(\tau s)^{2}+2 \zeta \tau s+1} \\
S(s) & =\frac{s^{2}\left(s+2 \omega_{c}\right)}{\left(s^{2}+\omega_{c} s+\omega_{c}^{2}\right)\left(s+\omega_{c}\right)} .
\end{aligned}
$$

Fig. 2 shows the frequency characteristics of disturbance reduction $S(s)$ and observation noise reduction $1-S(s)$ of the function (16).

Then, Fig. 1(a) can be transformed to Fig. 1(c). It shows that the two-degree-of-freedom control system includes an equivalent system to the disturbance observer. In a design of disturbance observer, one of the important issues is how to express the disturbance generation on the augmented state equation. In other words, this problem is equivalent to how much to consider the order of the disturbance. However, from the viewpoint of disturbance observer design, it is difficult to consider disturbance regulation on the output. On the other hand, in the design of two-degree-of-freedom control system, this problem is equivalent to a consideration of the order of parameter $S(s)$. And then, the condition of disturbance regulation on the output has been given as the condition for parameter $S(s)$. Besides, two-degreeof-freedom control type realization receives less influence of operation time delay when the system is realized as a digital system, compared with disturbance observer type.

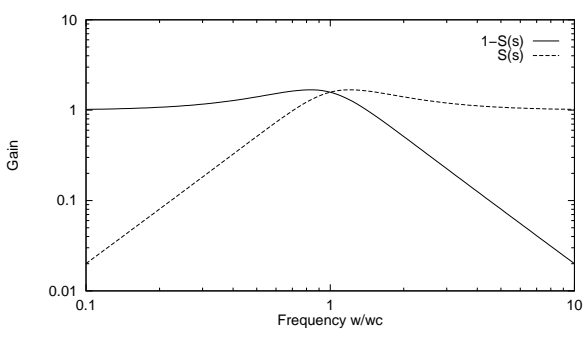

Fig. 2. Frequency characteristics of $S(s)$ and $1-S(s)$.

\section{Introduction of Sliding Mode}

A disturbance which cannot be completely compensated by the linear controller remains in the two-degreeof-freedom control system. Then, we introduce a sliding mode control to suppress this disturbance suppression error.

The feature of sliding mode control is that the robustness for fluctuation of plant parameters can be obtained by the simple control logic. However, in the case of conventional sliding mode control, if the load torque is larger than the dither signal, the sliding mode does not exist and stability is not assured. Therefore, the dither needs to be established large enough, but it causes a large chattering which has bad influence on the mechanism. In order to reduce this chattering, the authors have proposed to compensate some part of the load torque by the disturbance observer [13]-[15]. Then, the proposed system does not need the large dither. In this paper, the proposed system is constructed by replacing some parts of the linear controller of two-degree-of-freedom control system, which decides command input response $G_{y r}(s)$, with a sliding mode controller (Fig. 3(a)). The remaining linear controller has the same effect as the disturbance observer.

Fig. 3(a) can be transformed equivalently to Fig. 3(b). In Fig. 3(b), after a disturbance being reduced by the linear controller, the plant system can be described as the following error system:

$$
\begin{aligned}
\frac{d}{d t}\left[\begin{array}{c}
e \\
\dot{e}
\end{array}\right]= & {\left[\begin{array}{cc}
0 & 1 \\
0 & -B_{n} / J_{n}
\end{array}\right]\left[\begin{array}{l}
e \\
\dot{e}
\end{array}\right] } \\
& -\left[\begin{array}{c}
0 \\
K_{n} / J_{n}
\end{array}\right] u+\left[\begin{array}{c}
0 \\
K_{n} / J_{n}
\end{array}\right] \Delta d^{\prime}
\end{aligned}
$$

where

$$
\begin{aligned}
e & \equiv \theta_{\text {ref }}-\theta \\
\dot{e} & \equiv \dot{\theta}_{\text {ref }}-\dot{\theta} \\
\Delta d^{\prime} & \equiv S * d^{\prime} \\
d & =T_{l} / K .
\end{aligned}
$$

Here, $\Delta d^{\prime}$ is defined as the disturbance suppression error by the linear controller and $S$ is defined in time domain as impulse response of transfer function $S(s)$. The operator $*$ expresses the convolution.

The sliding mode control can be applied to this system. In this proposed method, the control input is determined 


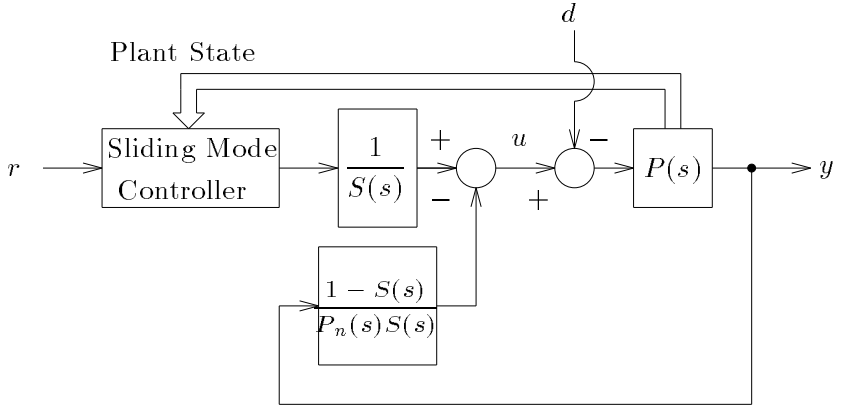

(a)

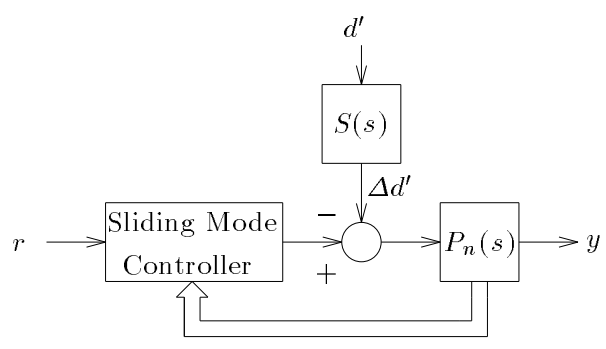

Plant State

(b)

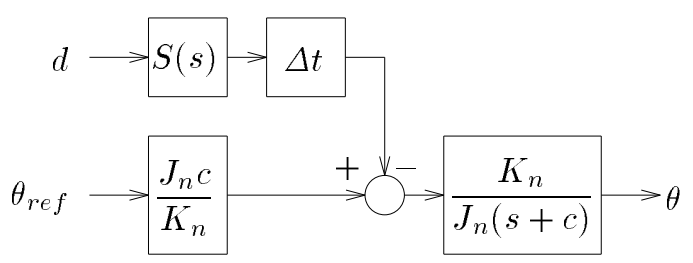

(c)

Fig. 3. Proposed system. (a) System configuration. (b) Equivalent block diagram. (c) Equivalent block diagram under sliding mode (dc motor control system).

by the following logic, in order to direct the trajectories in the state space toward the sliding line $s=0$.

$$
u=\psi e+K_{f} \operatorname{sgn}(\mathrm{s})
$$

where

$$
\begin{aligned}
& \mathrm{s}=c e+\dot{e} \\
& \psi= \begin{cases}\alpha & \text { if } \mathrm{s} e>0 \\
\beta & \text { if } \mathrm{se}<0 .\end{cases}
\end{aligned}
$$

Here, the parameter $K_{f}$ is a dither signal which compensate the disturbance.

The parameters of sliding mode controller can be determined from the stable condition of the global system. The next function is defined as a Lyapunov function [16]

$$
V=\frac{1}{2} \mathrm{~s}^{2}
$$

Using (17)-(25), the derivative can be calculated as

$$
\dot{V}=-\left(\frac{B_{n}}{J_{n}}-c\right) \mathrm{s}^{2}-\left(\frac{K_{n}}{J_{n}} \psi-c\left(\frac{B_{n}}{J_{n}}-c\right)\right) \mathrm{se}
$$
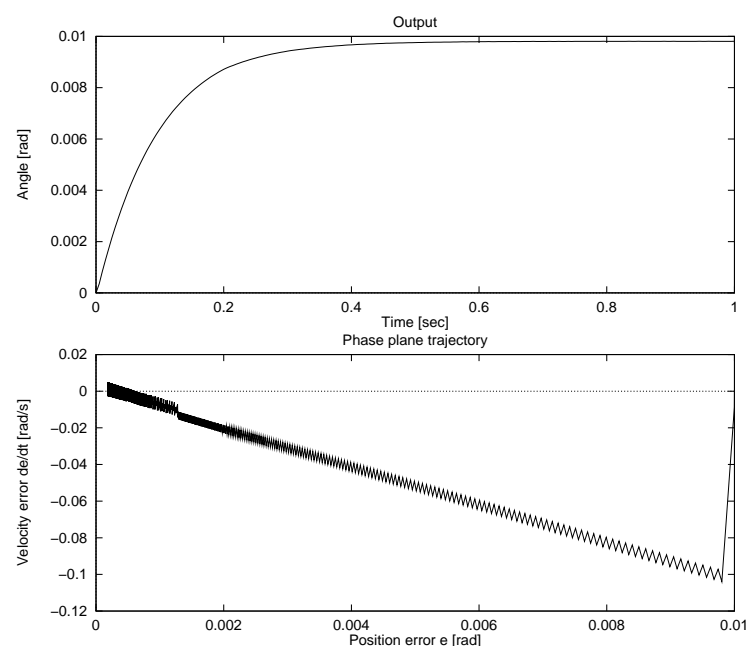

Fig. 4. Shift of trajectories. (Simulation)

$$
-\frac{K_{n}}{J_{n}}\left(K_{f}-\Delta d^{\prime} \operatorname{sgn}(\mathrm{s})\right)|\mathrm{s}|
$$

When $V$ is positive definite and $\dot{V}$ is negative, the system is stable. Thus, the following conditions are obtained:

$$
\begin{aligned}
c & \leq \frac{B_{n}}{J_{n}} \\
\psi & =\left\{\begin{array}{rr}
\alpha & >\frac{J_{n}}{K_{n}}\left(\frac{B_{n}}{J_{n}}-c\right) c \text { if } \mathrm{se}>0 \\
\beta & <\frac{J_{n}}{K_{n}}\left(\frac{B_{n}}{J_{n}}-c\right) c \text { if } \mathrm{se}<0
\end{array}\right. \\
K_{f} & >\left|\Delta d^{\prime}\right|
\end{aligned}
$$

where $(27),(28)$ and (29) correspond to the condition of existence of sliding mode and reachability to the sliding line. Against very large step function type disturbance, inequation (29) cannot be satisfied temporarily. However, due to the linear controller, $\left|\Delta d^{\prime}\right|$ becomes smaller and the state variables will come back to the sliding line within a finite time.

The above proof of system stability has an assumption that the linear controller in equation (29) is stable. Thus, it is clear that the total system becomes stable only if the inner linear system is stable.

\section{Disturbance Response}

When a plant system is controlled by sliding mode control, the plant dynamics are bound to the sliding line [6], [7]. Under the ideal sliding mode, the trajectories are completely equal to the equation of sliding line. However, if there is a switching delay $\Delta t$, the trajectories slightly shift from the sliding line in proportion to the magnitude of disturbance. In the case of system (17), the equation of the trajectories is modified as follows from (46):

$$
c e+\dot{e}=\frac{\Delta t K_{n}}{J_{n}} \Delta d^{\prime} .
$$

The detail of the derivation is shown in appendix. Fig. 4 shows such a phenomenon, where $\Delta d^{\prime}=$ 


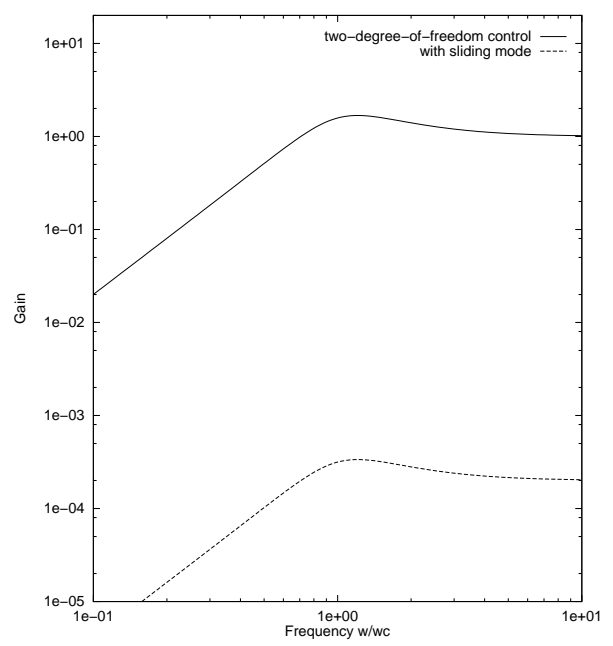

Fig. 5. Disturbance suppression effects in frequency domain.

$0.26[\mathrm{~V}]=0.5[\mathrm{~N} \cdot \mathrm{m}]$ is inflicted at $t>0.2[\mathrm{~s}]$, and the shifted trajectories can be observed.

After all, the following equation is derived as a global dynamics under the sliding mode.

$$
\theta(s)=\frac{c}{s+c} \theta_{r e f}(s)-\frac{K_{n}}{J_{n}(s+c)} \Delta t S(s) d^{\prime}(s) .
$$

Here, we assume $\dot{\theta}_{\text {ref }}=0$ because we do not deal with Continuous-Path servo problem.

Fig. 3(c) shows the equivalent block diagram. Thus, if the switching time $\Delta t$ is taken short enough, the disturbance gets more insensitive over all frequency domain (shown in Fig. 5 at $\Delta t=200[\mu \mathrm{s}]$ ). Now, we can set the sampling time less short than $1 \mathrm{~m} \mathrm{~s}$ using DSP, so that the disturbance suppression error can be reduced below $10^{-3}$.

However, from inequation (29), only when the disturbance suppression error $\Delta d^{\prime}$ is smaller than the dither $K_{f}$, the sliding mode exists and the equivalent block diagram Fig. 3(c) is realized. Fig. 6 shows the various order of disturbance which satisfies this condition. If the disturbance is located within this domain, the equivalent block diagram Fig. 3(c) is completely accomplished and the disturbance suppression becomes very strong, as Fig. 5 .

If a very large disturbance beyond the domain is imposed, some part of the disturbance suppression depends on only linear controller. Thus, the disturbance becoming the larger, the disturbance response becomes similar to one of pure two-degree-of-freedom control.

\section{Simulation Results}

The proposed control schemes are applied for position control of DC servo motor. Controllers are realized in digital system with 1 step delay at $200-\mu$ s sampling time. In the linear controller, the disturbance cut-off frequency $\omega_{c}$ is selected to $300[\mathrm{rad} / \mathrm{s}]$ on (16). The parameters of the

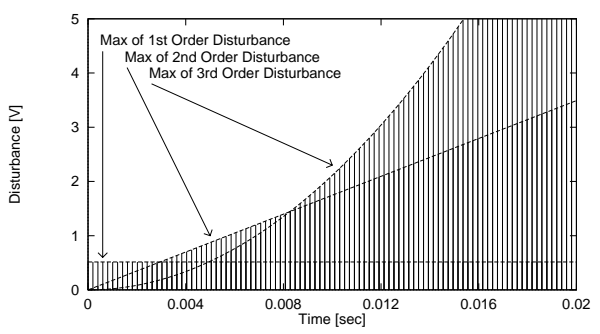

Fig. 6. Suppressible disturbance in time domain by sliding mode.

sliding mode controller are set as follows:

$$
\begin{array}{rlrl}
c & =10 & {[\mathrm{rad} / \mathrm{s}]} \\
\alpha & =120 \\
\beta & =0 \\
K_{f} & =0.51 \quad[\mathrm{~V}] \quad(=1.0[\mathrm{~N} \cdot \mathrm{m}]) .
\end{array}
$$

In order to compare with pure two-degree-of-freedom control, the parameter $G_{y r}(s)$ is set as $\tau=75[\mathrm{~ms}]$ and $\zeta=0.9$ on $(15)$.

In the simulations, two kind of disturbances are imposed.

\section{A. Step Type Disturbance}

Simulation results of the step response under very large step function type disturbances $(5.1[\mathrm{~V}](=10[\mathrm{~N} \cdot \mathrm{m}]), 0.2[\mathrm{~s}]$ $<\mathrm{t}<0.7[\mathrm{~s}])$ are shown in Fig. 7(a) and Fig. 7(b). The imposed disturbance in Fig. 7(a), Fig. 7(b) is 10 times lager than the dither. Therefore, the improvement of disturbance suppression by sliding mode cannot be observed obviously. The disturbance here is out of range in Fig. 6 .

\section{B. Sinusoidal Type Disturbance}

The step response under very large sinusoidal disturbance (amplitude $5.1[\mathrm{~V}](=10[\mathrm{~N} \cdot \mathrm{m}])$, frequency $50[\mathrm{rad} / \mathrm{s}]$, $\mathrm{t}>0.2[\mathrm{~s}])$ are shown in Fig. $7(\mathrm{c})$, Fig. $7(\mathrm{~d})$. The lowfrequency disturbance imposed in Fig. 7(c), Fig. $7(\mathrm{~d})$ is located within the domain of suppressible disturbance in Fig. 6 , which can be compensated by sliding mode. Thus, in this case, the proposed control scheme has very stronger disturbance compensation than pure two-degree-of-freedom control.

\section{Experimental Results}

In the experiments, DSP (NEC: $\mu$ PD77230, 32-b floating point) are used at $200[\mu \mathrm{s}]$ sampling time, and the $1 / 100$ gear ratio DC servo motor is driven by a $20-\mathrm{kHz}$ switching frequency MOSFET chopper. The pulse counter generates 1000 pulse per roll on the motor shaft (it becomes 1000000 pulse/roll on the geared shaft), and the speed is detected through 12-b A/D converter by a tachometer. In the experiments, two kind of artificial disturbances were inflicted by adding disturbance voltage to the input.

Experiments of the step response under very large step function type disturbances $(5.1[\mathrm{~V}](=10[\mathrm{~N} \cdot \mathrm{m}]), 0.2[\mathrm{~s}]<\mathrm{t}$ $<0.7[\mathrm{~s}]$ ) are shown in Fig. 8(a), Fig. 8(b). In Fig. 8(b), some overshoot appeared, probably because of the influence of the switching delay with rotor inductance. 

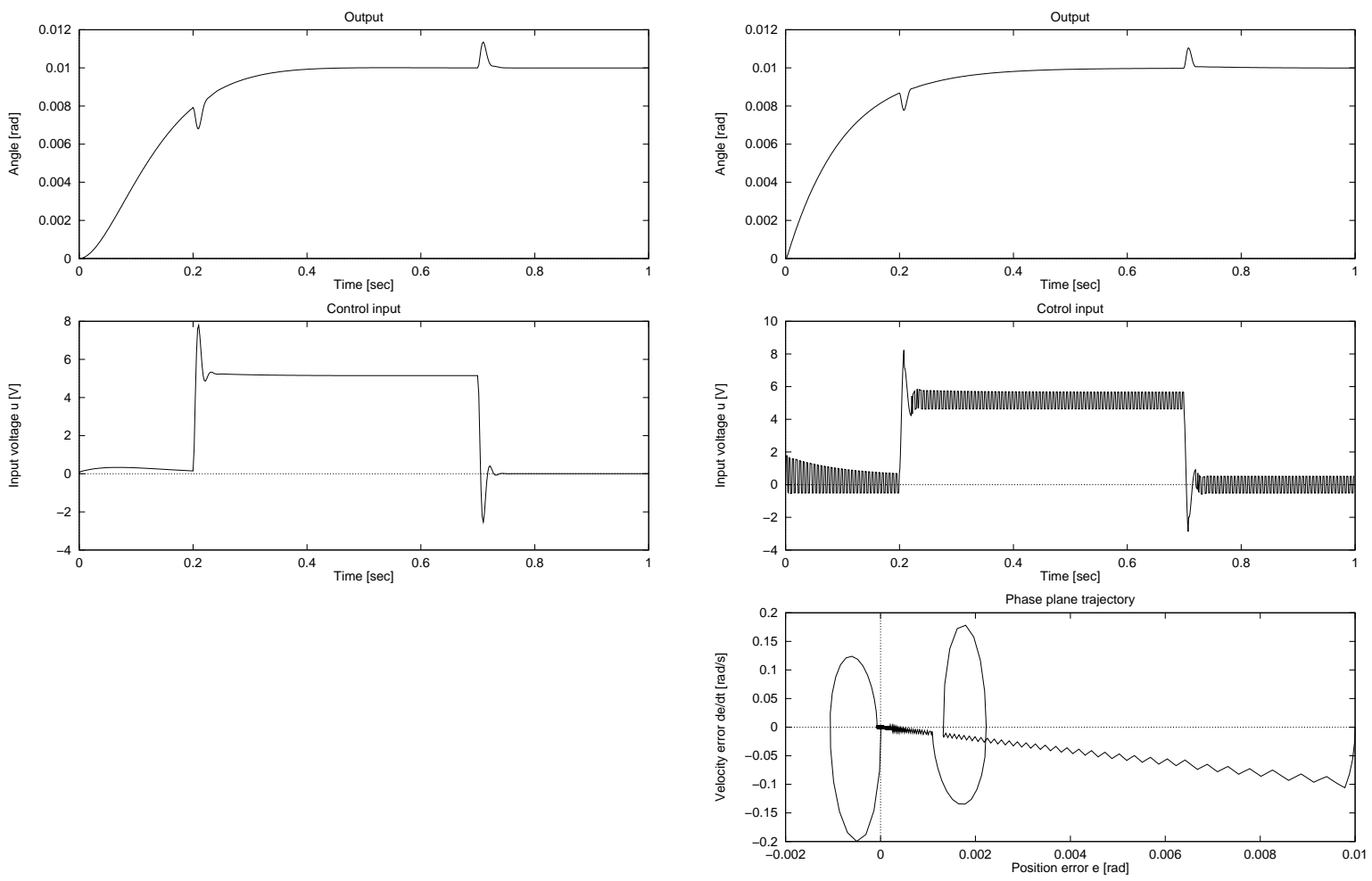

(a)

(b)

Fig. 7. Simulation result of step response under various disturbances. (a) Two-degree-of-freedom control under step disturbance. (b) Proposed control under step disturbance. (c) Two-degree-of-freedom control under sinusoidal disturbance. (d) Proposed control under sinusoidal disturbance.

The step response under very large sinusoidal disturbance (amplitude $5.1[\mathrm{~V}](=10[\mathrm{~N} \cdot \mathrm{m}])$, frequency $50[\mathrm{rad} / \mathrm{s}]$, $\mathrm{t}>0.2[\mathrm{~s}])$ are shown in Fig. 8(c), Fig. 8(d).

These results are almost similar to the simulation results.

\section{Robustness for Inertia Fluctuation}

The robustness for the inertia fluctuation are shown in Fig. 9, (b) of which is a step response when the plant inertia $J$ becomes 3 times larger than the nominal inertia $J_{n}$. There is almost no differences between the response (b) and the one without inertia fluctuation Fig. 9(a).

\section{Conclusion}

For almost all kinds of disturbances except high-speed ones, the proposed control system has very strong robustness. The reason is that the sensitivity of disturbance can be reduced in proportion to switching frequency of sliding mode. In other words, using the dither, the proposed system can compensate the remaining disturbance, which cannot be completely compensated by the linear controller. When we use a DSP and set the sampling time less short than $1 \mathrm{~ms}$, the proposed control can eliminate the disturbance suppression error more effectively to the order of $10^{-3}$.

In combination with sliding mode control, two-degree-offreedom control becomes more robust.

\section{APPENDIX}

In order to consider the disturbance response under the sliding mode, we assume that the conditions of existence of sliding mode are satisfied.

When the system is expressed as follows and the switching delay $\Delta t$ exists, the state transition in the neighborhood of sliding line becomes as Fig. 10.

$$
\dot{\boldsymbol{x}}= \begin{cases}\boldsymbol{f}^{+} & \text {if } \mathrm{s} \geq 0 \\ \boldsymbol{f}^{-} & \text {if } \mathrm{s} \leq 0 .\end{cases}
$$

At this time, the average fluctuation of state transition $\Delta \overline{\boldsymbol{x}}$ can be calculated as follows:

$$
\Delta \overline{\boldsymbol{x}}=\frac{1}{t_{\text {total }}} \int_{0}^{t_{\text {total }}} \boldsymbol{x}^{\text {real }}(t) d t-\frac{1}{t_{\text {total }}} \int_{0}^{t_{\text {total }}} \boldsymbol{x}^{\text {ideal }}(t) d t
$$

where

$$
t_{\text {total }}=\Delta t+\tau_{1}+\Delta t+\tau_{2}
$$

where definitions of the time interval $\tau_{1}$ and $\tau_{2}$ are shown in Fig. 10. 

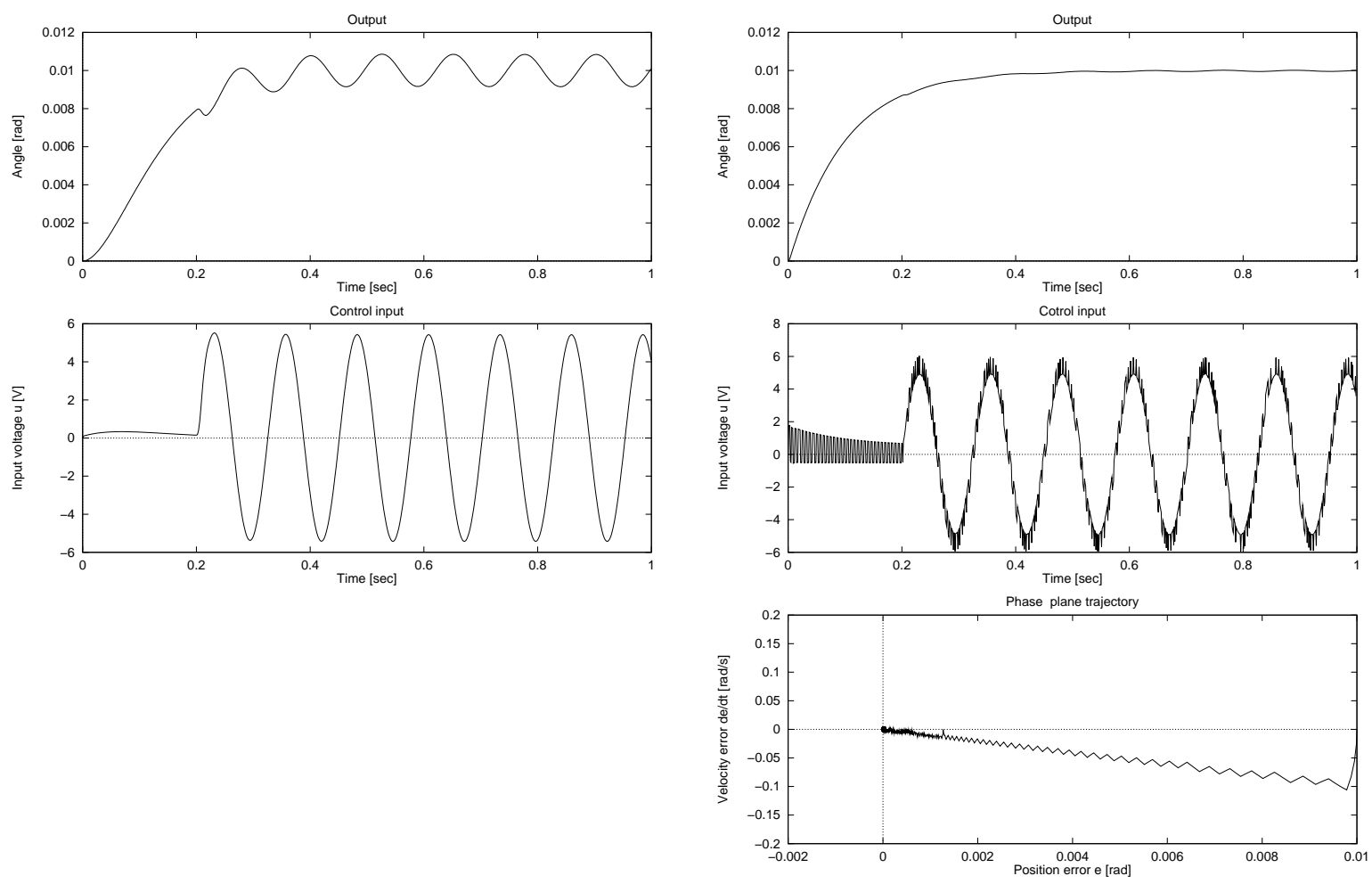

(c)

(d)

Fig. 7. (Cont.)

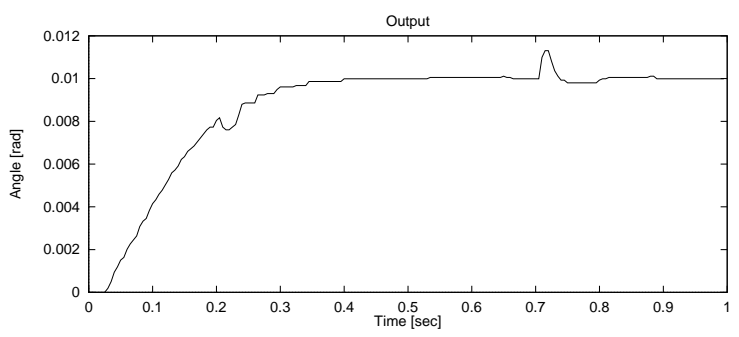

(a)

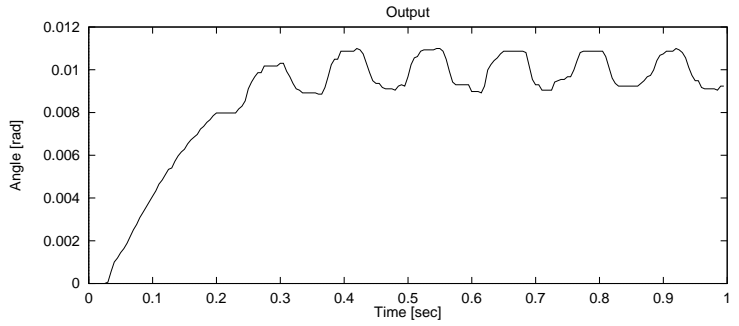

(c)

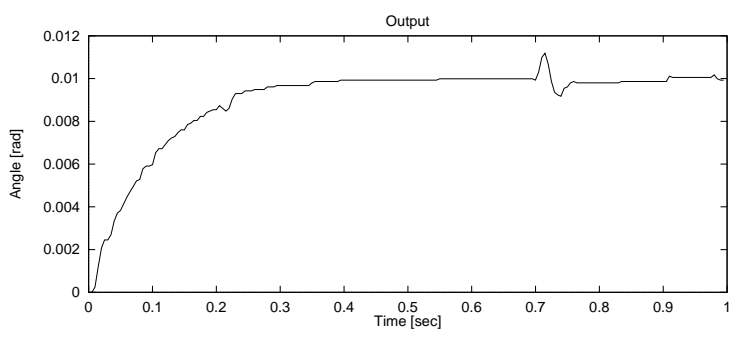

(b)

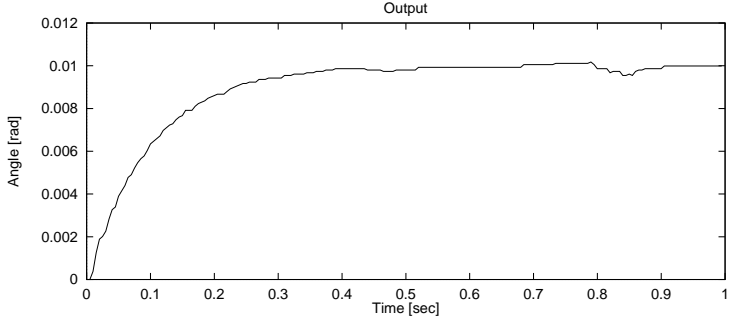

(d)

Fig. 8. Experimental result of step response under various disturbances. (a) Two-degree-of-freedom control under step disturbance. (b) Proposed control under step disturbance. (c) Two-degree-of-freedom control under sinusoidal disturbance. (d) Proposed control under sinusoidal disturbance. 


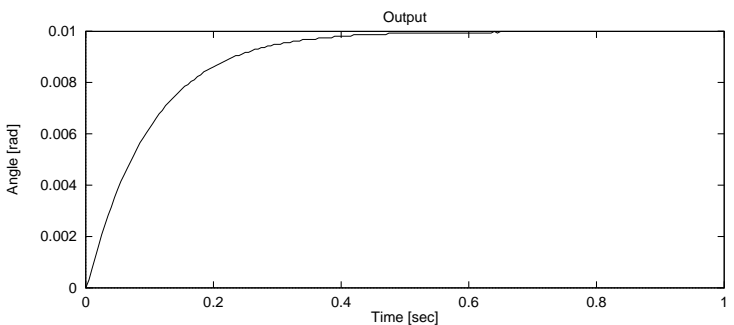

(a)

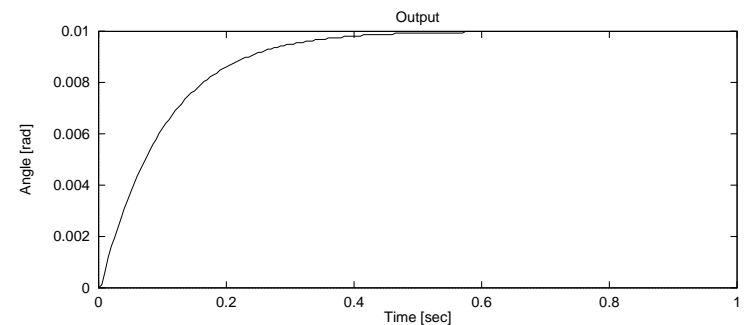

(b)

Fig. 9. Robustness for inertia fluctuation (Simulation). (a) $J=J_{n}$. (b) $J=3 \times J_{n}$.

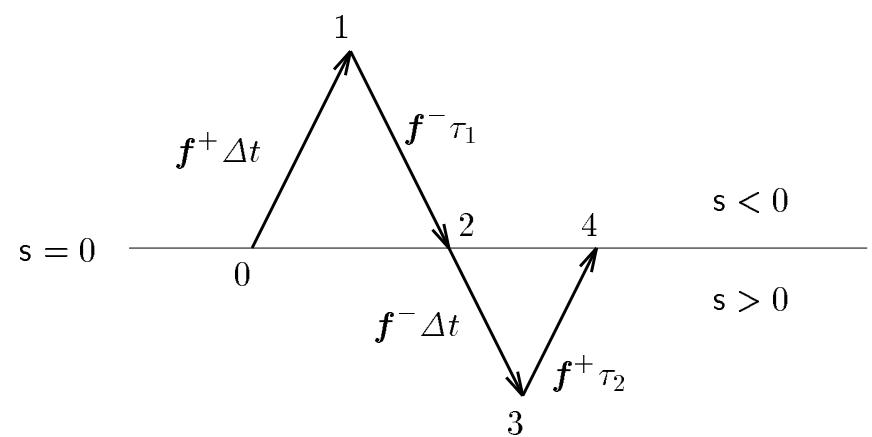

Fig. 10. State transition in delay $\Delta t$.

From Fig. 10, $\boldsymbol{x}^{\text {real }}(t)$, the state transition from the point $(0)$ with delay, becomes as follows:

$$
\begin{aligned}
& \boldsymbol{x}^{\text {real }}(t) \begin{array}{r}
\boldsymbol{f}^{+} t \quad \\
\boldsymbol{f}^{+} \Delta t+\boldsymbol{f}^{-}(t-\Delta t) \\
\quad \text { if } 0 t<t<\Delta t+\tau_{1} \\
\boldsymbol{f}^{+} \Delta t+\boldsymbol{f}^{-} \tau_{1}+\boldsymbol{f}^{-}\left[t-\left(\Delta t+\tau_{1}\right)\right] \\
\quad \text { if } \Delta t+\tau_{1}<t<\Delta t+\tau_{1}+\Delta t \\
\boldsymbol{f}^{+} \Delta t+\boldsymbol{f}^{-} \tau_{1}+\boldsymbol{f}^{-} \Delta t+\boldsymbol{f}^{+}\left[t-\left(\Delta t+\tau_{1}+\Delta t\right)\right] \\
\quad \text { if } \Delta t+\tau_{1}+\Delta t<t<\Delta t+\tau_{1}+\Delta t+\tau_{2} .
\end{array}
\end{aligned}
$$

Then, we can obtain the value of $\tau_{1}$ by calculating the distance between the point(1) and the sliding line in Fig. 10 as follows:

$$
\operatorname{grad} \mathrm{s} \cdot \boldsymbol{f}^{+} \Delta t=-\operatorname{grad} \mathrm{s} \cdot \boldsymbol{f}^{-} \tau_{1} \text {. }
$$

About the point(3), we can also obtain a similar equation.

$$
\operatorname{grad} \mathrm{s} \cdot \boldsymbol{f}^{-} \Delta t=-\operatorname{grad} \mathrm{s} \cdot \boldsymbol{f}^{+} \tau_{2} \text {. }
$$

On the other hand, $\boldsymbol{x}^{\text {ideal }}(t)$ expresses the ideal state transition from the point $(0)$ as follows:

$$
\boldsymbol{x}^{i d e a l}(t)=\boldsymbol{f}^{\text {ideal }} t
$$

where

$$
\boldsymbol{f}^{\text {ideal }}=\mu \boldsymbol{f}^{+}+(1-\mu) \boldsymbol{f}^{-} .
$$

Here, $\mu$ is determined by the condition $\operatorname{grad} \mathrm{s} \cdot \boldsymbol{f}^{\text {ideal }}=0$ in which the hyper plane $s=0$ and the vector (39) come in contact with each other (Filippov method [8]). Then, (39) becomes as

$\boldsymbol{f}^{\text {ideal }}=\frac{\operatorname{grad} \mathrm{s} \cdot \boldsymbol{f}^{-}}{\operatorname{grad} \mathrm{s} \cdot\left(\boldsymbol{f}^{-}-\boldsymbol{f}^{+}\right)} \boldsymbol{f}^{+}-\frac{\operatorname{grad} \mathrm{s} \cdot \boldsymbol{f}^{+}}{\operatorname{grad} \mathrm{s} \cdot\left(\boldsymbol{f}^{-}-\boldsymbol{f}^{+}\right)} \boldsymbol{f}^{-}$.

Using these equations (34)-(38) and (40), we can calculate $\Delta \overline{\boldsymbol{x}}$ from the equation (33) and get the average fluctuation of state transition $\Delta \overline{\boldsymbol{x}}$ as follows:

$$
\Delta \overline{\boldsymbol{x}}=\frac{\Delta t \operatorname{grad~s} \cdot\left(\boldsymbol{f}^{+}+\boldsymbol{f}^{-}\right)}{2 \operatorname{grads} \cdot\left(\boldsymbol{f}^{+}-\boldsymbol{f}^{-}\right)}\left(\boldsymbol{f}^{+}-\boldsymbol{f}^{-}\right) .
$$

Therefore, the equation of trajectories is modified as $\mathrm{s}(\boldsymbol{x}-$ $\Delta \overline{\boldsymbol{x}})=0$ from ideal dynamics of hyper plane $\mathrm{s}(\boldsymbol{x})=0$.

When the plant system is expressed as the following linear system and the switched control input is the following state feedback:

$$
\begin{aligned}
\dot{\boldsymbol{x}} & =\boldsymbol{A} \boldsymbol{x}+\boldsymbol{B} \boldsymbol{u}+\boldsymbol{\Gamma} \boldsymbol{d} \\
\boldsymbol{u} & = \begin{cases}\boldsymbol{F}^{+} \boldsymbol{x} & \text { if } \mathrm{s} \geq 0 \\
\boldsymbol{F}^{-} \boldsymbol{x} & \text { if } \mathrm{s} \leq 0\end{cases}
\end{aligned}
$$

where

$$
\mathrm{s}=\boldsymbol{C} \boldsymbol{x}
$$

from (41), the modified system dynamics $\boldsymbol{C}(\boldsymbol{x}-\Delta \overline{\boldsymbol{x}})=0$ becomes

$$
\boldsymbol{C}\left(1-\Delta t\left(\boldsymbol{A}+\boldsymbol{B} \frac{\boldsymbol{F}^{+}+\boldsymbol{F}^{-}}{2}\right)\right) \boldsymbol{x}=\Delta t \boldsymbol{C} \boldsymbol{\Gamma} \boldsymbol{d} .
$$

If the switching delay $\Delta t$ is selected enough short and $1 \ll$ $\Delta t\left(\boldsymbol{A}+\boldsymbol{B}\left(\boldsymbol{F}^{+}+\boldsymbol{F}^{-}\right) / 2\right)$, the next approximation is realized

$$
\boldsymbol{C x}=\Delta t \boldsymbol{C r \boldsymbol { d }} .
$$

This is equivalent to $(30)$.

\section{REFERENCES}

[1] T. Umeno and Y. Hori, "Design of Robust Servosystems Based on the Parametrization of Two Degrees of Freedom Control Systems", Trans. IEE Japan, vol. 109-D, no. 11, pp. 825-832, 1989 (in Japanese).

[2] D. C. Youla, H. A. Jabr, and J. J. Bongiorno, JR., "Modern Wiener-Hopf Design of Optimal Controllers - Part II: The Multivariable Case", IEEE Trans. Automat. Contr., vol. AC-21, pp. 319-338, 1976.

[3] C. A. Desoer and C. L. Gustafson, "Algebraic Theory of Linear Multivariable Feedback Systems", IEEE Trans. Automat. Contr., vol. AC-29, pp. 909-917, 1984. 
[4] T. Sugie and T. Yoshikawa, "General Solution of Robust Tracking Problem in Two-Degree-of-Freedom Control Systems", IEEE Trans. Automat. Contr., vol. AC-31, pp. 552-554, 1986.

[5] K. Matsumoto, T. Suzuki, S. Sangwongwanich, and S. Okuma, "Internal Structure of Two-Degree-of-Freedom Controller and a Design Method for Free Parameter of Compensator", Trans. IEE Japan, vol. 133-D, no. 6, pp. 768-777, 1993 (in Japanese).

[6] V. I. Utkin, "Variable Structure Systems with Sliding Modes", IEEE Trans. on Automat. Contr., vol. AC-22, pp. 212-222, 1977.

[7] H. Hashimoto, "Motion Control Using VSS", J. Robotics Society of Japan, vol. 11, no. 4, pp. 515-520, 1993 (in Japanese).

[8] J. J. Slotine and W. Li: Applied Nonlinear Control, Englewood Cliffs, NJ: Prentice Hall, 1991.

[9] K. Ohishi, M. Nakao, K. Ohnishi, and K. Miyachi, "Microprocessor-Controlled DC Motor for Load-Insensitive Position Servo System", IEEE Trans. Ind. Electron., vol. IE-34, pp. 44-49, 1987.

[10] K. Ohnishi, "Robust Motion Control by Disturbance Observer", J. Robotics Society of Japan, vol. 11, no. 4, pp. 486-493, 1993 (in Japanese).

[11] T. Murakami, F. Yu, and K. Ohnishi, "Torque Sensorless Control in Multidegree-of-Freedom Manipulator", IEEE Trans. Ind. Electron., vol. IE-40, pp. 259-265, 1993.

[12] T. Umeno, T. Kaneko, and Y. Hori, "Robust Servosystem Design with Two Degrees of Freedom and its Application to Novel Motion Control of Robot Manipulators", IEEE Trans. Ind. Electron., vol. IE-40, pp. 473-485, 1993.

[13] A. Kawamura, K. Miura, and T. Ishizawa, "Observer Based Sliding Mode CP Control of Two Axis SCARA Robot", in Proc. IEEE Int. Workshop on Advanced Motion Control, 1990, pp. $197-202$.

[14] H. Itoh and A. Kawamura, "Optimal Design Procedure of Disturbance Observer Based Sliding Mode CP Control For Two Axis SCARA Robot", in Proc. IEEE IECON'91, 1991, pp. 11851190 .

[15] A. Kawamura, H. Itoh, and K. Sakamoto, "Chattering Reduction of Disturbance Observer Based Sliding Mode Control", IEEE Trans. Ind. Appl., vol. 30, pp. 456-461, 1994.

[16] Y. Fujimoto and A. Kawamura, "Robust Servo-system Based on Two-Degree-of-Freedom Control with Sliding Mode", in Proc. IEEE IECON'93, 1993, pp. 85-90.

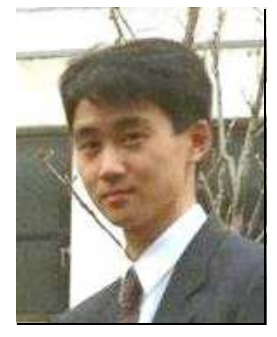

Yasutaka Fujimoto was born in Kanagawa, Japan, in 1971. He received B.S. degree in electrical engineering from Yokohama National University, Yokohama, Japan, in 1993.

Since 1993, he has been a postgraduate student in the Department of Electrical and Computer Engineering, Yokohama National University. His research interests include motion control, digital control, and robotics.

Mr. Fujimoto is a menber of the IEE of Japan and the Robotics Society of Japan.

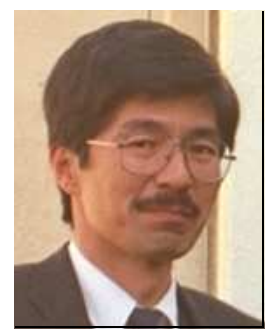

Atsuo Kawamura was born in Yamaguchi, Japan, in 1953. He received the B.S., M.S., and Ph.D. degrees in electrical engineering from the University of Tokyo, Japan, in 1976, 1978, and 1981, respectively.

In 1981 he joined the Department of Electrical and Computer Engineering, University of Missouri, Columbia, as a Postdoctoral Fellow, and was an Assistant Professor there from 1983 to 1986 . In 1986 , he joined the faculty of Yokohama National University, Yokohama, Japan, where he is presently an Associate Professor in the Department of Electrical and Computer Engineering. His interests are in power electronics, digital control, ultrasonic actuators, and robotics.

Dr. Kawamura is a member of the IEE of Japan, the Robotics Society of Japan, the Institute of Electronics Information and Communication Engineers, and the Society of Instrument and Control Engineering. He is a recipient of the IEEE Industry Applications Society Transactions Prize Paper Award in 1988. 